\title{
BTS guidelines for the initial outpatient management of pulmonary embolism: there's no place like home
}

\author{
Luke S Howard
}

\begin{abstract}
This article provides an overview of the highlights and key points of the new BTS guideline for the initial outpatient management of pulmonary embolism.
\end{abstract}

Nowadays, with modern therapies and better understanding of risk, we are able to shorten hospital stays for many elective and non-elective hospital admissions. Ambulatory care centres and protocols have been on the increase for the last decade with the aim of treating more patients with acute conditions at home. It is now well established that patients with deep vein thrombosis, by and large, can be managed at home, and it is evident from the medical literature that the same trend is occurring in patients with acute pulmonary embolism.

In the 2014 European Society of Cardiology Pulmonary Embolism guidelines, ${ }^{1}$ patients with a low risk according to a Pulmonary Embolism Severity Index (PESI) or simplified PESI (sPESI) could potentially be considered for early outpatient management, but in-depth evidence review and guidance was not provided. Neither the National Institute for Health and Care Excellence ${ }^{2}$ guideline in 2012, nor its update in 2015, issued recommendations regarding outpatient management of pulmonary embolism. Pulmonary embolism is well known by the medical profession and the public to be potentially fatal; therefore, if we are to manage patients presenting with this condition at home, robust standardised guidelines and pathways must be in place to reassure healthcare commissioners, hospitals, the medical profession and, finally, patients and carers. The British Thoracic Society Standards of Care Committee thus agreed in 2014 to commission guidelines on the topic of initial outpatient management of pulmonary embolism. These are published

National Pulmonary Hypertension Service, Hammersmith Hospital, Imperial College Healthcare NHS Trust, London, London, UK

Correspondence to Dr Luke S Howard, National Pulmonary Hypertension Service, Hammersmith Hospital, Imperial College Healthcare NHS Trust, London W12 OHS, UK; I.howard@imperial.ac.uk as a supplement in this issue of Thorax, ${ }^{3}$ with a summary of recommendations and good practice points available in $B M J$ Open Respiratory Research. ${ }^{4}$

In view of the evidence demonstrating safe outcomes in selected patients with pulmonary embolism being treated at home, the guideline makes recommendations that haemodynamically-stable patients should be risk assessed for suitability for outpatient management pathways where a robust system is in place for follow-up and monitoring and appropriate safeguards are in place. Most of the studies are prospective cohorts of patients selected for their low-risk characteristics, but in the largest randomised, controlled study of inpatient versus outpatient management, good levels of patient satisfaction were achieved in both the inpatient and outpatient arms. ${ }^{5}$ Of note, many of the patients treated as outpatients would have preferred to have been kept in the hospital longer. We should not, therefore, assume that outpatient management of pulmonary embolism is what patients want, despite delivering economic benefits.

Scoring systems for severity of pulmonary embolism and checklists of exclusion criteria for outpatient management were extracted from the literature. Those which were supported by the most evidence were the PESI, sPESI and Hestia criteria. Only PESI was used in a randomised controlled study of inpatient versus outpatient management. ${ }^{5}$ The potential advantage of the PESI system is that there are five categories of risk, enabling clinicians to riskstratify beyond suitability for outpatient management; however, a further layer of exclusion criteria are required to ensure safe discharge, for example, the absence of pain requiring opiates. In contrast, the Hestia criteria were designed as a bespoke tool to be used in isolation for selection of outpatient management of pulmonary embolism. ${ }^{6}$ sPESI has the overall advantage of being the simplest score. ${ }^{7}$

There was no evidence supporting the need for additional biomarker measurement, such as troponin or brain natiuretic peptide (BNP), or assessment of right ventricular function by CT scanning or echocardiography, meaning that decisions regarding admission versus immediate discharge can be made with clinical risk scores at the bedside. Where right ventricular dilatation is seen and reported on CT scanning, it is however recommended to use a biomarker measurement which, if negative, categorises the patient as low risk.

The evidence for the effectiveness of risk stratification comes only from studies of confirmed pulmonary embolism, as indeed does the majority of evidence for outpatient management. Akin to some deep vein thrombosis pathways, some institutions already choose to risk assess suspected pulmonary embolism and discharge patients home with empirical anticoagulation therapy, pending a diagnostic scan. The rationale for this seems relatively straightforward, given that risk stratification tools do not require biomarkers or imaging. Two UK studies adopting this approach with bespoke risk stratification provided some evidence for the guideline committee to support a strategy of outpatient management of suspected pulmonary embolism ${ }^{89}$ with the proviso that imaging is performed within 24 hours and patients have senior level review.

Most of the data generated for outpatient management of pulmonary embolism have been derived from studies undertaken in the low molecular weight heparin/warfarin era, yet the direct oral anticoagulants are increasingly used in the treatment of pulmonary embolism. These agents lend themselves well to outpatient management, in particular those not requiring a heparin lead in. One potential risk related to their ease of use compared with heparin/warfarin is the lack of ongoing patient contact that is required, and for this reason, as well as good practice, it is strongly advised that patients are provided with high-quality verbal and written information regarding symptoms and signs of recurrence, major bleeding and other complications. Moreover, we recommend that patients should have a formal review, either over telephone or face to face, at least once during the first week after discharge. Patients should be provided with an appropriate point of contact, both in and out of hours. It is important that treatment of the acute phase of pulmonary embolism feeds into robust long-term follow-up for issues such as duration of anticoagulation and monitoring of complications.

Specific circumstances were also considered in order to make the guideline applicable across a broad range of conditions. Although evidence is lacking, we recommend pregnant and 
postpartum women should also be considered for outpatient care pathways, but here clinical risk scores cannot be routinely applied, and consultant review as well as discussion with maternity services is needed. Patients with cancer, those over the age of 80 years and those with multiple comorbidities may never qualify for outpatient management when risk scores are used, yet it may be entirely appropriate for these patients to be sent home. It was therefore recognised by the group that senior clinical review could potentially sanction outpatient management, where standard criteria are not met. Conversely, clinicians should feel empowered to overrule these guidelines when deciding to keep patient in the hospital, despite apparently meeting outpatient management criteria. This situation may arise in young patients with a large clot burden, who are able to compensate physiologically, yet where there may be concern of decompensation, for example, in the presence of significant proximal deep vein thrombosis.

It is hoped that these focused guidelines on outpatient management of pulmonary embolism will bring to an end unnecessary variation to risk stratification and management of pulmonary embolism in the ambulatory care setting by providing standardised protocols and recommendations. A further aim is that better standardisation of management at the front door may lead to improved risk stratification of sicker patients with pulmonary embolism, as well as facilitating long-term follow-up, in terms of screening for complications and deciding duration of anticoagulation.

Contributors LSH chaired the guideline group, and as the author of this article, was responsible for drafting and final approval of the article.

Competing interests $\mathrm{LSH}$ received funding from Bayer PLC, BTG PLC and BMS.

Patient consent Not required.

Provenance and peer review Not commissioned; internally peer reviewed.

(C) Article author(s) (or their employer(s) unless otherwise stated in the text of the article) 2018. All rights reserved. No commercial use is permitted unless otherwise expressly granted.

Check for updates

To cite Howard LS. Thorax 2018;73:607-608.

Received 6 February 2018

Revised 16 May 2018

Accepted 21 May 2018

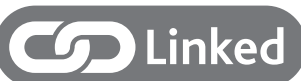

- http://dx.doi.org/10.1136/thoraxjnl-2018-211539
Thorax 2018;73:607-608.

doi:10.1136/thoraxjnl-2018-211646

\section{REFERENCES}

1 Konstantinides SV, Torbicki A, Agnelli G, et al. 2014 ESC guidelines on the diagnosis and management of acute pulmonary embolism. Eur Heart J 2014;35:3033-69.

2 NICE. Venous thromboembolic diseases: the management of venous thromboembolic diseases and the role of thrombophilia testing, 2012. Updated 2015.

3 Howard LS, Barden S, Condliffe R, et al. British Thoracic Society Guideline for the initial outpatient management of pulmonary embolism (PE).Thorax 2018; 73(S2). doi:10.1136/thoraxinl-2018-211539.

4 Howard LS, Barden S, Condliffe R, et al. British Thoracic Society Guideline for the initial outpatient management of pulmonary embolism. BMJ Open Resp Res 2018; 5: e000281.

5 Aujesky D, Roy PM, Verschuren F, et al. Outpatient versus inpatient treatment for patients with acute pulmonary embolism: an international, open-label, randomised, non-inferiority trial. Lancet 2011;378:41-8

6 Zondag W, Mos IC, Creemers-Schild D, et al. Outpatient treatment in patients with acute pulmonary embolism: the Hestia Study. J Thromb Haemost 2011;9:1500-7.

7 Jiménez D, Aujesky D, Moores L, et al. Simplification of the pulmonary embolism severity index for prognostication in patients with acute symptomatic pulmonary embolism. Arch Intern Med 2010;170:1383-9.

8 Vali Y, Ladwa R, Bailie E, et al. Investigating and managing suspected pulmonary embolism in an outpatient setting: the Leicester experience. Thorax 2015;70:291-3.

9 Hogg K, Dawson D, Mackway-Jones K. Outpatient diagnosis of pulmonary embolism: the MIOPED (Manchester Investigation Of Pulmonary Embolism Diagnosis) study. Emerg Med J 2006;23:123-7. 